\title{
Beta decay radiation signature from neutron-rich gamma-ray bursts?
}

\author{
Soebur Razzaque and Peter Mészáros \\ Department of Astronomy \& Astrophysics, Department of Physics, Pennsylvania State University, \\ University Park, PA 16802
}

\begin{abstract}
Core collapse of massive stars and binary neutron stars or black hole-neutron star binary mergers are likely progenitors of long and short duration gamma-ray bursts respectively. Neutronized material in the former and neutron star material in the latter are ejected by the central engine implying a neutron-rich jet outflow. A free neutron, however, beta decays to a proton, an electron (beta) and an anti-neutrino in about fifteen minutes in its rest frame. Sudden creation of a relativistic electron is accompanied by radiation with unique temporal and spectral signature. We calculate here this radiation signature collectively emitted by all beta decay electrons from neutron-rich outflow. Detection of this signature may thus provide strong evidence for not only neutron but also for proton content in the relativistic gamma-ray burst jets.
\end{abstract}

Subject headings: gamma rays: bursts—gamma rays: theory—ISM: jets and outflows—radiation mechanisms: non thermal

\section{Introduction}

The constituents of relativistic gamma-ray burst (GRB) jets are not known completely as yet. The observed MeV energy $\gamma$-rays are most likely radiated by energetic electrons which are shock accelerated by internal collisions of successively ejected materials by the GRB central engine. In this widely accepted fireball shock model, protons along with electrons are needed to be present in the GRB jet to explain the observed rapid variability of $\gamma$-rays and the GRB afterglow. Free neutrons are speculated to be present in the GRB jet (Derishev, Kocharovsky \& Kocharovsky 1999a) which may be a common feature (Beloborodov 2003b) following the ejection of neutronized core material in case of a long burst which are most likely created by core collapse of massive stars, a mechanism similar to that of the supernovae of type Ib/c or type II. Recent observations of several short burst afterglows suggest a common relativistic jet feature in both the long and short bursts. In the latter case, neutron star material may also feed the jet with free neutrons.

Observational signatures (both electromagnetic and neutrino) of a neutron component in the GRB jets have been discussed by many authors, in the early fireball evolution phase (Derishev, Kocharovsky \& Kocharovsky 1999a; Bahcall \& Mészáros 2000; Mészáros \& Rees 2000; Razzaque \& Mészáros 2006) and later in the internal shocks or afterglow phase (Derishev, Kocharovsky \& Kocharovsky 1999b; Pruet \& Dalal 2002; Beloborodov 2003a; Fan \& Wei 2004; Fan, Zhang \& Wei 2005; Dermer \& Atoyan 2006). 
However, most of these predictions depend heavily on the jet model parameters and interpretation of data may be difficult.

In this paper we discuss a generic signature of neutron-rich jets, namely the radiation emitted by relativistic electrons created by neutron beta decay: $n \rightarrow p e^{-} \bar{\nu}_{e}$ in the GRB jets. This radiation signature should be ubiquitiously present in the electromagnetic signal detectable from all GRBs if the jet contains free neutrons. We also find that the signal strength do not vary rapidly for reasonable ratios of the neutrons to protons in the jet and/or other jet parameters. Thus detection or non-detection of this signature may serve as a powerful discriminator between GRB jets with and without a neutron component.

We discuss the neutron-rich jet models of long and short bursts in Section 2, beta decay electron energy and number distribution in Section 3 and radiation signature in Section 4. We discuss our results and detection prospects of beta decay radiation in Section 5 .

\section{Neutron-rich jet models}

The typical parameters we use for long and short GRB jets are listed in Table 1. The baryon loading parameter (also known as the dimensionless entropy) is defined as the total energy to mass flow ratio in the GRB jet: $\eta=L / \dot{M} c^{2}$. The total mass outflow rate in the jet, ignoring the electron mass $\left(m_{e} \ll m_{p}\right)$ and negligible thermal energy, is $\dot{M} \simeq 4 \pi r^{2} c\left(1+\xi_{o}\right) n_{p}^{\prime} m_{p}$. Here $\xi_{o}=n_{n}^{\prime} / n_{p}^{\prime}$ is the initial neutron to proton number density ratio in the jet comoving frame. The outflow starts at a radius $R_{O}$ which is a few times the Schwarzschild radius $r_{g}=2 G M_{\mathrm{bh}} / c^{2}$ of a solar mass (few solar mass) black hole for a short (long) GRB.

Electrons, protons and neutrons are coupled to thermal radiation in the expanding jet outflow as long as the Compton scattering time scales $t_{\mathrm{Th}}^{\prime} \simeq\left(n_{p}^{\prime} \sigma_{\mathrm{Th}} c\right)^{-1}$ and elastic $n$ - $p$ scattering time scale $t_{n p}^{\prime} \simeq$ $\left(n_{p}^{\prime} \sigma_{n p} c\right)^{-1}$ are shorter than the plasma expansion time $t_{\exp }^{\prime} \simeq r / c \Gamma(r)$ in the jet comoving frame (see, e.g., Rossi, Beloborodov \& Rees (2005) for a detailed description of the neutron-rich jet dynamics). Here, $\Gamma(r) \propto r / R_{o}$ is the bulk Lorentz factor of the expanding jet outflow and the elastic $n-p$ scattering crosssection $\sigma_{n p} \approx \sigma_{\mathrm{Th}} / 20$, the Thomson cross-section. The final value of $\Gamma$, after the expansion phase is over, depends on the value of $\eta$. For sufficiently high value of $\eta$, the neutrons may decouple from the outflow (Derishev, Kocharovsky \& Kocharovsky 1999a; Bahcall \& Mészáros 2000). The corresponding critical $\eta$ value for $n$ - $p$ decoupling, from the condition $t_{n p}^{\prime}=t_{\exp }^{\prime}$, is

$$
\eta_{n p} \simeq\left[\frac{L \sigma_{n p}}{4 \pi R_{o} m_{p} c^{3}\left(1+\xi_{o}\right)}\right]^{1 / 4} \approx\left\{\begin{array}{l}
264\left(L_{52} / R_{o, 7}\right)^{1 / 4}\left(1+\xi_{o, 1}\right)^{-1 / 4} \\
148\left(L_{50} / R_{o, 6}\right)^{1 / 4}\left(1+\xi_{o, 1}\right)^{-1 / 4}
\end{array}\right.
$$

Here we have used $\xi_{o}=10 \xi_{o, 1}$. The final bulk Lorentz factor of the neutrons in the jet outflow is

$$
\Gamma_{n, f}= \begin{cases}R_{n p} / R_{o} ; & \eta>\eta_{n p} \\ \Gamma_{p, f} \approx \eta ; & \eta<\eta_{n p}\end{cases}
$$

where $R_{n p}$ is the $n$ - $p$ decoupling radius where the nuclear scattering optical depth $\tau_{n p}^{\prime} \simeq n_{p}^{\prime} \sigma_{n p} R_{n p} / \Gamma\left(R_{n p}\right)=$ 
1 and is given by

$$
R_{n p} \simeq \eta_{n p}\left(\frac{\eta_{n p}}{\eta}\right)^{1 / 3} \approx\left\{\begin{array}{l}
2.5 \times 10^{9}\left(L_{52} R_{o, 7}^{2} / \eta_{2.5}\right)^{1 / 3}\left(1+\xi_{o, 1}\right)^{-1 / 3} \mathrm{~cm} \\
1.2 \times 10^{8}\left(L_{50} R_{o, 6}^{2} / \eta_{2.5}\right)^{1 / 3}\left(1+\xi_{o, 1}\right)^{-1 / 3} \mathrm{~cm}
\end{array}\right.
$$

Note that for $\eta>\eta_{n p}$ the final Lorentz factor for the electron, proton and radiation outflow $\Gamma_{p, f}>\Gamma_{n, f}$ [see, e.g., Rossi, Beloborodov \& Rees (2005)]. In any case $\Gamma_{n, f} \lesssim \eta$, always.

The kinetic luminosity of the freely coasting neutron outflow in the GRB jet is given by

$$
L_{n, \mathrm{k}}=L \frac{\Gamma_{n, f}}{\eta} \frac{\xi_{o}}{1+\xi_{o}}
$$

Initially, at time $t \sim 0$, the neutron volume number density in the outflow, in the jet comoving frame, is

$$
n_{n, o}^{\prime}=\frac{L_{n, \mathrm{k}}}{4 \pi r^{2} \beta_{n} m_{n} c^{3} \Gamma_{n, f}^{2}}
$$

Here $\beta_{n} \sim 1$. However, this decreases exponentially with time as neutrons decay (beta decay) which we discuss next.

\section{Beta decay}

A free neutron has a mean lifetime $\tau_{\beta}=886.7 \mathrm{~s}$ in its rest frame. This corresponds to a mean decay radius $R_{\beta}=c \tau_{\beta} \Gamma_{n, f}$ for a neutron at rest in the Lorentz boosted outflow. The volume number density of beta decay electrons (or protons or neutrinos) in the outflow evolves with time as $n_{e}^{\prime}(t)=n_{n, o}^{\prime}(1-$ $\left.\exp \left[-r(t) / R_{\beta}\right]\right)$ and the total number of beta decay electrons at a time $t$ is $N_{e}^{\prime}(t)=4 \pi r^{2}(t) n_{e}^{\prime}(t) c t$ with no energy injection at later time $t \gtrsim 0$. We denote the variables in the frame comoving with the neutron outflow with primes from now on. The relationship between the observed time and neutron outflow radius is $r(t)=2 c t \Gamma_{n, f}^{2} /(1+z)$.

For our purpose we are interested in the total number of freshly created electrons at a time $t$ which we define approximately, using Equation (5), as

$$
\mathcal{N}_{e}^{\prime}(t) \simeq \frac{\min \left[t, t_{90}\right] L_{n, \mathrm{k}}}{m_{n} c^{2} \Gamma_{n, f}^{2}} \times \begin{cases}1-e^{-r(t) / R_{\beta}} ; & r(t) \leq R_{\beta} \\ e^{-r(t) / R_{\beta}} ; & r(t)>R_{\beta}\end{cases}
$$

The validity of this approximation depends on the fast rise and exponential decay of the function with time. Here $t_{90} L_{n, \mathrm{k}}$ is the total energy carried by the neutron outflow in case of a GRB central engine active for a time $t_{90}$. Note that in case of complete decoupling of the neutron component from the rest of the outflow for $\Gamma_{n, f} \ll \Gamma_{p, f}$, the observed GRB may result from the leading proton outflow with $t_{90}$ duration and the lagging neutron component may have a different time scale. However, we ignore this possibility for the sake of simplicity. 
The total energy released in a beta decay is the difference between the rest mass energies of the initial and final particles: $Q=m_{n} c^{2}-\left(m_{p}+m_{e}+m_{\nu}\right) c^{2}=0.782-m_{\nu} c^{2} \mathrm{MeV}$ [see, e.g., Krane (1987)]. This energy is shared by the kinetic energies of the electron $\left(T_{e}\right)$, neutrino $\left(T_{\nu}\right)$ and proton $\left(T_{p}\right)$ in the decaying neutron's rest frame. With a negligible neutrino mass and tiny proton recoil energy: $T_{p} \sim 0.3 \mathrm{keV}$ (because of its large mass), the maximum electron's kinetic energy is $T_{e, \max } \simeq Q$ for $T_{\nu} \rightarrow 0$. The shape of the kinetic energy spectrum of beta decay electrons is given by

$$
N_{e}\left(T_{e}\right)=\sqrt{T_{e}^{2}+2 T_{e} m_{e} c^{2}}\left(Q-T_{e}\right)^{2}\left(T_{e}+m_{e} c^{2}\right)
$$

which vanishes for $T_{e}=0$ and $Q$.

The kinetic energy of the electrons in Equation (6) is then distributed according to Equation (7) and we write a normalized kinetic energy distribution of beta decay electrons at an observed time $t$, in the jet outflow, as

$$
\frac{d \mathcal{N}_{e}^{\prime}}{d T_{e}^{\prime}}(t)=\frac{\mathcal{N}_{e}^{\prime}(t) N_{e}^{\prime}\left(T_{e}^{\prime}\right)}{\int_{0}^{Q} N_{e}^{\prime}\left(T_{e}^{\prime}\right) d T_{e}^{\prime}}
$$

We verify this expression by numerically integrating the right hand side over $T_{e}^{\prime}$ in the range $0-Q$ and comparing with Equation (6) for different $t$. Figure 1 shows the distribution in Equation (8) plotted for different parameters for long and short GRB jets at a time $t_{\beta}=\tau_{\beta} / 2 \Gamma_{n, f}$ (assuming $z \sim 0$ ) when the total number of freshly created beta decay electrons in Equation (6) is the maximum. Note that $\mathcal{N}_{e}^{\prime}$ increases with $\xi_{o}$, however, it is not directly proportional since $\Gamma_{n, f}$ is not directly proportional to $\xi_{o}$ [see Equation (2)]. We have listed different values of $t_{\beta}$ and $\Gamma_{n, f}$ in Table 2 for different choice of $\eta$ and $\xi_{o}$ in case of long and short burst curves plotted in Figure 1.

\section{Radiation spectrum and lightcurve}

Beta decay electrons are created suddenly with a peak kinetic energy of $0.25 \mathrm{MeV}$ in the neutron's rest frame. These electrons may be thought to be created initially at rest and then accelerated to a constant high speed $v=c \beta_{e}$ within a short time interval $\tau \sim \hbar c / E_{e}$, following the uncertainty principle. Here

$E_{e}=T_{e}+m_{e} c^{2}$ is the total energy of the electron. Rapidly accelerating electrons then emit radiation (often called inner bremsstrahlung in the literature; see Petrosian \& Ramaty (1972) for an early astrophysical application) similar to the bremsstrahlung. The radiation spectrum (total energy radiated per unit frequency interval) is also flat as bremsstrahlung and is given by (Jackson 1999)

$$
\frac{d I}{d \omega}=\frac{q^{2}}{\pi c}\left[\frac{1}{\beta_{e}} \ln \left(\frac{1+\beta_{e}}{1-\beta_{e}}\right)-2\right]
$$

where the electron's speed (with momentum $p_{e}$ ) is

$$
\beta_{e}=\frac{p_{e} c}{E_{e}}=\frac{\sqrt{T_{e}^{2}+2 T_{e} m_{e} c^{2}}}{T_{e}+m_{e} c^{2}} \approx 0.74
$$




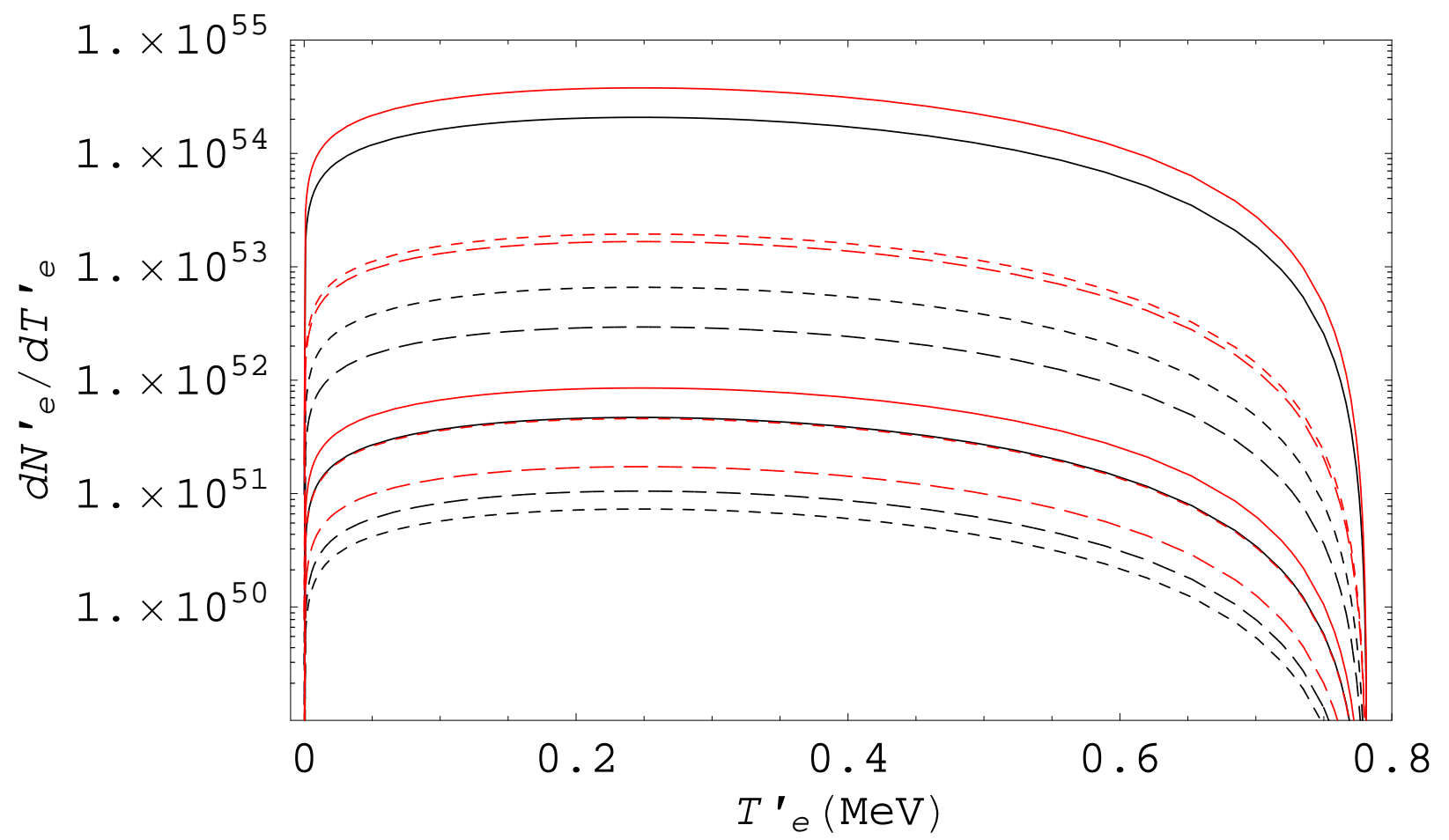

Fig. 1.- Kinetic energy distribution [Equation (8)] of beta decay electrons normalized to the total number of electrons [Equation (6)] in the GRB jet comoving frame at an observed time $t_{\beta}=\tau_{\beta} / 2 \Gamma_{n, f}$ for which $r(t)=R_{\beta}$ or when the initial number of neutrons have decayed by a factor $1 / e$. The upper (lower) set of six curves correspond to long (short) GRBs. The solid, dashed and dotted-dash pair of curves correspond to $\eta=100,316$ and 500 with $\xi_{o}=10\left(\xi_{o}=1\right)$ for the upper (lower) curve in each pair. The other parameters for both the long and short GRBs are listed in Table 1 . The calculated values of $\Gamma_{n, f}$ and $t_{\beta}$ for all curves are listed in Table 2. 
The last number is for electrons of peak kinetic energy. Note that the spectrum defined in Equation (9) is proportional to the more familiar notation $F_{\nu}$.

The maximum energy of the emitted photons from beta decay electrons is $\hbar \omega_{\max } \lesssim E_{e}$ following the condition $\omega \tau \lesssim 1$, although a full quantum mechanical treatment is necessary to evaluate the precise value. Semi-classically an electron loses a small fraction of its energy given by integrating Equation (9) over $\omega$ as

$$
\frac{E_{\gamma}}{E_{e}}=\frac{q^{2}}{\pi \hbar c}\left[\frac{1}{\beta_{e}} \ln \left(\frac{1+\beta_{e}}{1-\beta_{e}}\right)-2\right] \approx 0.001
$$

Again, the last number is for an electron of peak kinetic energy $0.25 \mathrm{MeV}$. However, the collective radiation energy from all beta decay electrons in a GRB jet is large. For an observed GRB, the beta decay radiation is beamed along the line of sight of an observer because of the relativistic bulk motion of the jet even though beta decay electrons do not have any particular angular orientation and the radiation is emitted along the electron's directon in the jet comoving frame.

We write the total energy radiated per unit frequency by all beta decay electrons at a time $t$ in Equation (8) as would be measured by an observer as

$$
\begin{aligned}
\frac{d \mathcal{I}}{d \omega}(t) & =\frac{\Gamma_{n, f}}{(1+z)} \int_{0}^{Q} \frac{d \mathcal{N}_{e}^{\prime}}{d T_{e}^{\prime}}(t) \frac{d I^{\prime}}{d \omega^{\prime}} d T_{e}^{\prime} \\
& =\frac{q^{2} \Gamma_{n, f}}{\pi c(1+z)} \int_{0}^{Q} \frac{d \mathcal{N}_{e}^{\prime}}{d T_{e}^{\prime}}(t)\left[\frac{1}{\beta_{e}^{\prime}} \ln \left(\frac{1+\beta_{e}^{\prime}}{1-\beta_{e}^{\prime}}\right)-2\right] d T_{e}^{\prime}
\end{aligned}
$$

which is flat in frequency. The corresponding total radiation energy emitted over all frequencies at a time $t$, by integrating Equation (12) over $0 \lesssim \hbar \omega \lesssim\left(T_{e}^{\prime}+m_{e} c^{2}\right) \Gamma_{n, f} /(1+z)$, is

$$
\mathcal{E}_{\gamma, \text { tot }}(t) \simeq \frac{q^{2} \Gamma_{n, f}^{2}}{\pi \hbar c(1+z)^{2}} \int_{0}^{Q}\left(T_{e}^{\prime}+m_{e} c^{2}\right) \frac{d \mathcal{N}_{e}^{\prime}}{d T_{e}^{\prime}}(t)\left[\frac{1}{\beta_{e}^{\prime}} \ln \left(\frac{1+\beta_{e}^{\prime}}{1-\beta_{e}^{\prime}}\right)-2\right] d T_{e}^{\prime}
$$

We have plotted the total energy radiated by beta decay electrons in Figure 2 as function of observed time by numerically evaluating the integral in Equation (13) for different GRB model parameters used in Figure 1 and listed in Table 2. The total number of electrons at the peak kinetic energy plotted in Figure 1 times the total energy radiated by each of these electrons using Equation (11) roughly corresponds to the peak total energy plotted in Figure 2 after multiplying with the corresponding $\Gamma_{n, f}^{2}$ factor. The maximum observed photon energy in all cases is $\epsilon_{\gamma, \max } \approx 1.3 \Gamma_{n, f} /(1+z) \mathrm{MeV}$.

\section{Detection prospects and Discussion}

The highest energy photons carry most of the radiation energy plotted in Figure 2, since the beta decay radiation spectrum from Equation (12) is flat. The corresponding photon flux (energy received per unit area per unit time) on Earth, in a given energy band $\epsilon_{\gamma, l^{-}} \epsilon_{\gamma, u}$; is

$$
\mathcal{F}\left(\epsilon_{\gamma, l}-\epsilon_{\gamma, u}\right) \simeq \frac{1}{4 \pi d_{L}^{2}} \int_{\epsilon_{\gamma, l}}^{\epsilon_{\gamma, u}} \frac{d \mathcal{I}}{d \omega}(t)
$$


Table 1. Typical Gamma-Ray Burst Parameters

\begin{tabular}{lcc}
\hline \hline \multicolumn{1}{c}{ Parameter } & Long GRB & Short GRB \\
\hline $\begin{array}{l}\text { Total isotropic-equivalent } \\
\text { energy outflow }(L) \text { ergs/s }\end{array}$ & $10^{52} L_{52}$ & $10^{50} L_{50}$ \\
Burst duration $\left(t_{90}\right) \mathrm{s}$ & 10 & 1 \\
Redshift $(z)$ & 1 & 0.1 \\
Luminosity distance ${ }^{\mathrm{a}}\left(d_{L}\right) \mathrm{cm}$ & $10^{28} d_{L, 28}$ & $10^{27} d_{L, 27}$ \\
Baryon loading parameter $(\eta)$ & $316 \eta_{2.5}$ & $316 \eta_{2.5}$ \\
Initial outflow radius $\left(R_{o}\right) \mathrm{cm}$ & $10^{7} R_{o, 7}$ & $10^{6} R_{o, 6}$ \\
\hline
\end{tabular}

${ }^{a}$ Using typical cosmological parameters

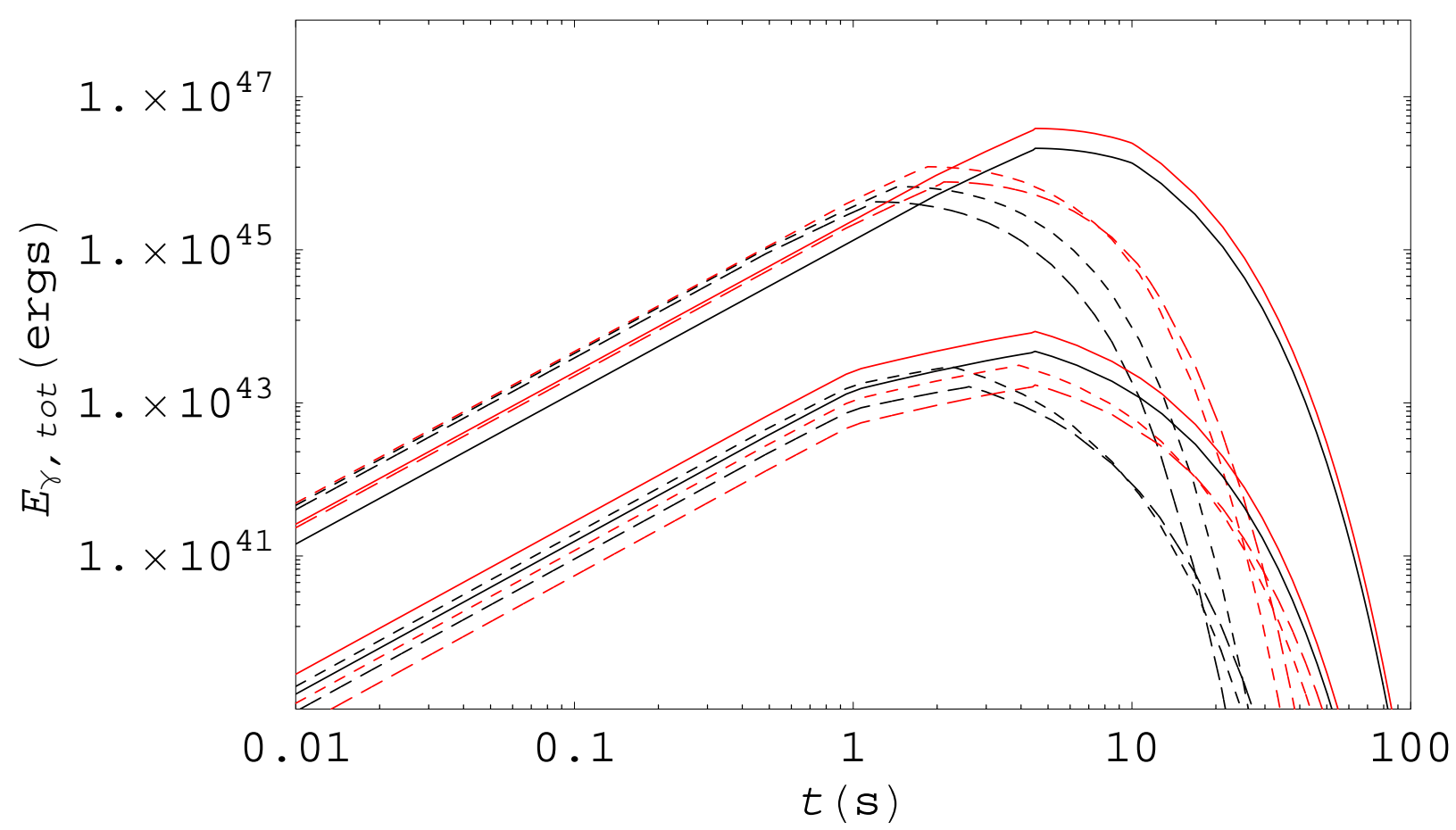

Fig. 2.- Total radiation energy emitted by beta decay electrons over all frequencies as function of time [Equation (13)]. The model parameters for the GRB jets for different curves are the same as in Figure 1 and listed in Table 2. The upper (lower) set of curves correspond to the long (short) bursts. Note that the signal is stronger and peaks later for $\Gamma_{n, f}=\eta$ cases as evidenced by the solid pair of curves in case of both long and short bursts. The dependence on $\xi_{o}$ is rather weak. 
Upcoming high energy $\gamma$-ray detectors such as the Large Area Telescope (LAT) on board the Gammaray Large Area Telescope (GLAST) are sensitive to photons in the energy range $20 \mathrm{MeV}-300 \mathrm{GeV}$. The threshold fluence for LAT is $\sim 4 \times 10^{-8} \mathrm{ergs} / \mathrm{cm}^{2}$ for a short integration time. This roughly corresponds to a few photons with energy $\gtrsim 0.1 \mathrm{GeV}$ collected within a few seconds in its average $5000 \mathrm{~cm}^{2}$ effective area over this energy range. We have listed in Table 2 the expected beta decay radiation flux at photon energies $\epsilon_{\gamma} \gtrsim 0.1 \mathrm{GeV}$, for different values of the relevant parameters. We used a luminosity distance $d_{L}=10^{27} d_{L, 27}$ $\mathrm{cm}$ corresponding to a redshift $z \sim 0.1$ for both the long and short bursts. This redshift is typical for short bursts, and is at the lower end for long bursts. (Observations indicate an occurrence rate of $\sim 0.25$ year $^{-1}$ for long bursts and 2-3 year ${ }^{-1}$ for short bursts in this redshift range).

However, the beta decay signal can be diluted by other types of emission in the same GRB, since the signal can coincide temporally with the usual prompt $\gamma$-ray emission phase. This is somewhat mitigated by the fact that the two signals have different spectra, and the beta decay signal has a smoother rise and quick decay, compared to the sometimes erratic and longer signal of the long burst prompt emission. The GRB afterglow emission occurs on an even longer time scale than the beta decay time scale $t_{\beta}$, and should not interfere with the latter. Hence we discuss the GRB prompt $\gamma$-ray emission as a source of background radiation below.

The observed isotropic-equivalent bolometric $\gamma$-ray luminsosity, $L_{\gamma}$, of a long (short) GRB is $\sim$ $10^{51} L_{\gamma, 51} \mathrm{ergs} / \mathrm{s}\left(\sim 10^{49} L_{\gamma, 49} \mathrm{ergs} / \mathrm{s}\right)$ which is mostly concentrated at the peak energy $\epsilon_{\gamma, \mathrm{pk}}=100 \epsilon_{\gamma,-4}$ $\mathrm{keV}$. In the fireball shock model, this energy corresponds to a fraction $\varepsilon_{e} \simeq L_{\gamma} / L \sim 0.1 \varepsilon_{e,-1}$ of the total energy converted by shock accelerated electrons. The luminosity at higher $\epsilon_{\gamma}$ decreases by a power-law, with index $2 \lesssim \alpha \lesssim 3$, following the synchrotron and/or inverse Compton (IC) radiation spectrum by a powerlaw distributed electrons. For $\alpha>2$, most of the energy in a given band $\epsilon_{\gamma, l^{-}} \epsilon_{\gamma, u}$ is carried by the low energy photons (unlike in the beta decay signal). The corresponding prompt (background) synchrotron/IC photon flux at Earth is approximately

$$
\begin{aligned}
F_{\text {prompt }}\left(\epsilon_{\gamma, l}-\epsilon_{\gamma, u}\right) & \simeq \frac{L_{\gamma}}{4 \pi d_{L}^{2}}\left(\frac{\epsilon_{\gamma, l}}{\epsilon_{\gamma, \mathrm{pk}}}\right)^{2-\alpha} ; \epsilon_{\gamma, l} \gtrsim \epsilon_{\gamma, \mathrm{pk}} \\
& \approx 8 \times 10^{-8}\left(\frac{\epsilon_{\gamma, l}}{0.1 \mathrm{GeV}}\right)^{-1} \frac{L_{\gamma, 51}}{d_{27}^{2} \epsilon_{\gamma,-4}} \mathrm{ergs} \mathrm{cm}^{-2} \mathrm{~s}^{-1} ; \alpha=3
\end{aligned}
$$

for a long GRB at $z \sim 0.1$. Thus, the detection of the beta decay radiation signature would be background limited in the case of long bursts with $t_{90} \gtrsim 2 \mathrm{~s}$, if the prompt spectrum extends to $\sim 0.1 \mathrm{GeV}$ with the above nominal spectrum. However, one can expect a sizable fraction of long GRBs where the prompt spectrum does not contain significant energy at $0.1 \mathrm{GeV}$, e.g. due to a spectral index which is steeper than the nominal value $\alpha=3$, or due to a fall-off in the spectrum [e.g., Preece, et al. (2000); also, as shown by Ryde (2004) that a fraction of long GRBs are dominated by a thermal-like component, with little or no emission above a few $\mathrm{MeV}]$. While data in the $\sim 0.1 \mathrm{GeV}$ range is currently still limited, one may conservatively estimate at $30 \%$ level the fraction of all long GRBs which do not have substantial emission at $0.1 \mathrm{GeV}$, and hence which contribute no background for the beta decay signal.

On the other hand, for short bursts, the beta decay signature peaks on timescales longer (see Figure 2) 
Table 2. Beta Decay Radiation Parameters

\begin{tabular}{|c|c|c|c|c|}
\hline Parameter & & $\Gamma_{n, f}$ & $t_{\beta}(\mathrm{s})$ & $\mathcal{F}^{\mathrm{a}}$ \\
\hline \multicolumn{5}{|c|}{ long GRB } \\
\hline \multirow[t]{2}{*}{$\eta=500$} & $\xi_{0}=1$ & 376 & 1.2 & 0.33 \\
\hline & $\xi_{o}=10$ & 213 & 2.1 & 0.61 \\
\hline \multirow[t]{2}{*}{$\eta=316$} & $\xi_{0}=1$ & 316 & 1.4 & 0.53 \\
\hline & $\xi_{o}=10$ & 248 & 1.8 & 0.97 \\
\hline \multirow[t]{2}{*}{$\eta=100$} & $\xi_{0}=1$ & 100 & 4.4 & 1.7 \\
\hline & $\xi_{o}=10$ & 100 & 4.4 & 3.1 \\
\hline \multicolumn{5}{|c|}{ short GRB } \\
\hline \multirow[t]{2}{*}{$\eta=500$} & $\xi_{0}=1$ & 174 & 2.5 & 2.6 \\
\hline & $\xi_{o}=10$ & 99 & 4.5 & 1.4 \\
\hline \multirow[t]{2}{*}{$\eta=316$} & $\xi_{0}=1$ & 203 & 2.2 & 2.4 \\
\hline & $\xi_{o}=10$ & 115 & 3.8 & 4.9 \\
\hline \multirow[t]{2}{*}{$\eta=100$} & $\xi_{0}=1$ & 100 & 4.4 & 3.8 \\
\hline & $\xi_{o}=10$ & 100 & 4.4 & 6.9 \\
\hline
\end{tabular}

ain units of $\left(\times 10^{-9}\right) \mathrm{ergs}^{-2} \mathrm{~s}^{-1}$ for long GRBs and in units of $\left(\times 10^{-12}\right) \mathrm{ergs}^{-2} \mathrm{~s}^{-1}$ for short GRBs 
than the prompt emission ( $t_{90} \lesssim 2 \mathrm{~s}$ ), and the beta decay signal is expected to be essentially free from a prompt emission background at $0.1 \mathrm{GeV}$.

One other source of background to consider is the diffuse extragalactic $\gamma$-ray background measured by EGRET (Sreekumar, et al. 1997). The corresponding photon number flux may be fitted in the $30 \mathrm{MeV}-120$ $\mathrm{GeV}$ energy range as $d N / d \epsilon_{\gamma} \approx 7.32 \times 10^{-9}\left(\epsilon_{\gamma} / 451 \mathrm{MeV}\right)^{-2.1} \mathrm{~cm}^{-2} \mathrm{~s}^{-1} \mathrm{sr}^{-1} \mathrm{MeV}^{-1}$. This background energy flux within $\sim 1^{\circ}$ angular resolution of LAT is $F_{\mathrm{bkg}} \approx 3 \times 10^{-14} \mathrm{ergs} \mathrm{cm}^{-2} \mathrm{~s}^{-1}$ for $\epsilon_{\gamma} \gtrsim 0.1 \mathrm{GeV}$ which is negligible compared to the beta decay flux listed in Table 2 or GRB prompt flux in Equation (15).

The predicted fluxes $\mathcal{F}(\gtrsim 0.1 \mathrm{GeV})$ in Table 2 were calculated for bursts of nominal (MeV) luminosities $L_{\gamma}=10^{51} \mathrm{erg} / \mathrm{s}$ (long bursts) and $L_{\gamma}=10^{49} \mathrm{erg} / \mathrm{s}$ (short bursts), at a redshift $z=0.1$. Multiplying these nominal fluxes by a typical average beta decay peak duration $t_{\beta} \sim 5 \mathrm{~s}$, and comparing to the LAT threshold sensitivity fluence $4 \times 10^{-8} \mathrm{ergs} / \mathrm{cm}^{2}$ at $\epsilon_{\gamma} \sim 0.1 \mathrm{GeV}$, one sees that for short bursts, even though it is background free, the signal is undetectable by the LAT. For long bursts, however, it may be possible to detect the beta decay radiation signature from nearby long bursts whose luminosity is ten times the average value. Bursts satisfying these criteria may represent $\sim 0.1-1 \%$ of a nominal total of 100 bursts per year detected by GLAST. This suggests that GLAST may be marginally able to detect such signals, and future generations of very energetic space-based $\gamma$-ray large area telescopes may be able to quantitatively explore this problem.

Non-thermal multi GeV energy neutrino and $\gamma$-ray emission from the $n$ - $p$ decoupling phase can indicate the presence of a neutron component in the GRB jets, for values of the dimensionless entropy $\eta \gtrsim \eta_{n p}$, which is typically an unknown parameter. On the other hand, the electromagnetic radiation signature discussed here is valid for all values of $\eta$. The evidence for a neutron component from the beta decay radiation signature would definitely imply the presence of protons in the GRB jet, since neutrons are coupled to them at least initially. Even though the converse is not true, the beta decay radiation signature may be an alternate way to explore the baryon loading in the GRB jets in the cases when high energy neutrinos from the internal or external shocks are absent (e.g., if the protons are not co-accelerated with electrons), or if too few are detected. The beta decay photon signature of neutron decay may thus be a valuable tool for investigating the particle acceleration process and possibly constrain the progenitors of Gamma-Ray Burst sources.

Research supported in part through NSF AST 0307376 and NASA NAG5-13286.

\section{REFERENCES}

Bahcall, J. N. and Mészáros, P. 2000, Phys. Rev. Lett., 85, 1362

Beloborodov, A. M. 2003, ApJ, 585, L19

Beloborodov, A. M. 2003, ApJ, 588, 931

Dermer, C. D. and Atoyan, A. 2006, astro-ph/0601142 
Derishev, E. V., Kocharovsky, V. V. and Kocharovsky, VI. V. 1999, ApJ, 521, 640

Derishev, E. V., Kocharovsky, V. V. and Kocharovsky, VI. V. 1999b, A\&A, 345, L51

Fan, Y. Z. and Wei, D. M. 2004, ApJ, 615, L69

Fan, Y. Z., Zhang, B. and Wei, D. M. 2005, ApJ, 628, L25

Jackson, J. D. 1999, Classical Electrodynamics, John Wiley \& Sons, 3rd edition, pp. 730

Krane, K. S. 1987, Introductory Nuclear Physics, John Wiley \& Sons, pp. 272

Mészáros, P. and Rees, M. J., 2000, ApJ, 541, L5

Petrosian, V. and Ramaty, R., 1972, ApJ, 173, L83

Preece, R. D., et al. 2000, ApJS, 126, 19

Pruet, J. and Dalal, N. 2002, ApJ, 573, 770

Razzaque, S. and Mészáros, P. 2006, ApJ (submitted), astro-ph/0601652

Rossi, E. M., Beloborodov, A. M. and Rees, M. J. 2005, astro-ph/0512495

Ryde, F. 2004, ApJ, 614, 827

Sreekumar, P., et al. 1997, ApJ, 494, 523 